http://dx.doi.org/10.12795/PH.1989.v04.i02.34

\title{
EL SUJETO DEL INFINITIVO ¿UN PROCESO ESTRUCTURAL?
}

\author{
Ángela Gracia Menéndez \\ Departamento de Filología Alemana \\ Universidad de Sevilla
}

En alemán e inglés las oraciones en las que la frase completiva contiene un infinitivo se caracterizan por la ausencia del sujeto de ese infinitivo, incluso en aquellos casos en los que el sujeto no es idéntico al sujeto del predicado en la frase. En español y francés las construcciones de infinitivo difieren de las alemanas, ya que siempre que el sujeto de la frase completiva es distinto al de la principal, se utiliza un subjuntivo.

Nuestra atención se centra en la descripción de las construcciones de infinitivo por la Gramática Generativa, con el objetivo de poner de relieve que la formulación de un principio, como el de «Mínima Distancia», basado únicamente en proximidad estructural no es válido ni adecuado para determinar el posible sujeto de la frase completiva de infinitivo.

Contrastaremos posteriormente la solución que ofrece Searle en Taxonomy of Illocutionary Acts por tratar de explicar el comportamiento sintáctico partiendo del significado del verbo, con función de predicado, que nosotros consideramos más adecuado.

La Gramática Generativa tuvo desde sus comienzos una gran repercusión en varias escuelas lingüísticas de las que destacamos aquí sólo la teoría de la lengua y la teoría gramatical.

El planteamiento de la Gramática Generativa constituye el primer enfoque significativo en el estudio de las construcciones de infinitivo abriendo nuevos caminos dentro de la disciplina lingüística, si tomamos la aparición del Cours de Linguistique Générale de Ferdinand de Saussure como punto de partida.

La importancia excesiva que Chomsky atribuye a aspectos formales en el estudio de la lengua se desprende ya de Syntactic Structures, en el que califica la Teoría Generatia de 
«completely formal and non-semantic»,

(1978:93)

posición que jamás llega a modificar sustancialmente, adoptando insuficiencias del Distribucionalismo americano, como es precisamente la preocupación exclusiva de la forma y la exclusión absoluta de la semántica.

Chomsky tiene una concepción de sintaxis errónea, como puede apreciarse en el 2. capítulo de Syntactic Structures titulado «Independence of Grammar», ya que evidentemente la sintaxis no puede ser estudiada independientemente del significado.

También las siguientes consideraciones ponen de manifiesto el papel predominante que atribuye a la sintaxis dentro de su teoría al considerarla autónoma:

«Despite the undeniable interest and importance of semantic and statistical studies of language, they appear to have no direct relevance to the problem of determining or characterizing the set of grammatical utterances, I think that we are forced to conclude that grammar is autonomous and independent of meaning,...»

Chomsky, (1978:17)

Chomsky utilizó en Aspecs of the Theory of Syntax (1965) las construcciones de infinitivo para justificar el concepto de una estructura profunda, en la que la NP (el sintagma nominal) realiza la función de sujeto del infinitivo, alegando que la estructura profunda aseguraría la interpretación semántica de la estructura superficial.

En su empeño de superar el método distribucionalista descriptivo de segmentación y clasificación, la Gramática Generativa introduce un componente explicativo en su teoría lingüística, repitiendo, sin embargo, errores del Distribucionalismo en cuanto a la limitación a consideraciones puramente estructurales, a ya la exclusión de la semántica.

Indudablemente ha sido un mérito de la Gramática Generativa destacar no sólo los límites de la metodología estructuralista, sino señalar también que la teoría estructuralista se reducía al conjunto de datos obtenido. Chomsky (1969:242).

El objetivo de Chomsky es «to construct a formalized general theory of linguistic stucture» Syntactic Structures $\left(1963^{3}: 5-6\right)$, teoría que consiste en la explicación de la competencia (19772:7). Chomsky parte de la idea de que todo hablante ha adquirido una gramática generativa, que, según él, es un sistema de reglas, que permite generar un número infinito de frases. Ese sistema de reglas consta de 3 componentes, el componente sintáctico, el fonológico y el semántico. El componente sintáctico tiene la función de generar una estructura profunda y una superficial para cada frase poniendo en correlación a ambas. La estructura profunda determina la interpretación de la frase, y la estructura superficial la interpretación fonológica, respectivamente (1969:30).

La necesidad de una estructura profunda radica en querer sustituir las funciones sintácticas tradicionales, como p. ej. sujeto por funciones «lógicas», es decir, semánticas, como puede ser «agente», partiendo de la gran mayoría de las frases, en las que en efecto el sujeto es el agente. Así se explica el empeño de especificar en la estructura profunda qué NP es el sujeto lógico del infinitivo. 
Consideramos inadecuada la distinción entre funciones sintácticas o gramaticales, por una parte, y semánticas o lógicas, por otra, arguyendo que en consideraciones dentro de un plano sintáctico, como la determinación de las funciones sintácticas, no deberían aplicarse relaciones semánticas como «agente», ya que sintácticas, no deberían aplicarse relaciones semánticas como «agente», ya que designan una relación externa a la lengua. Por consiguiente, la Gramática Generativa no se ha atenido a un formalismo estrictamente sintáctico o gramatical, sino que ha introducido indebidamente categorías semánticas, que a nuestro parecer es inadmisible, hablando de relaciones sintácticas.

Basándose en Aspects of the Theory of Syntax (1965), Rosenbaum formuló el principio de «Minimal Distance» en A Principle Governing Deletion in English Sentential Complementation (1970), con el fin de formular la transformación de elisión que no fue problematizada por Chomsky en Aspects. En él establece que aquella NP más próxima al infinitivo necesariamente es el sujeto del infinitivo, dentro del plano de la estructura profunda.

Así se asume para la frase (1)

(1) I persuaded a specialist to examine John.

la estructura profunda (2)

(2) I -persuaded -a especialist -a specialist will examine John.

Con el principio de mínima distancia, Rosenbaum incluso pensó haber descubierto un principio de la Gramática Universal que permitiría explicar al igual oraciones relativas y construcciones de gerundio. Efectivamente este principio funcionó, pero tan sólo en aquella clase de verbos que más tarde son denominados «object control verbs», como to ask, to want, etc.

En vez de corregir el principio, la Gramática Generativa prefirió mantenerlo, pero clasificando aquellos verbos que no correspondían con el principio establecido como «excepciones», como es el caso de to promise, que fácilmente se podía explicar por el significado. Fue precisamente el semántico generativo Jackendoff, quien se opuso al principio, alegando el significado de promise:

«This principle, if correct, would be a very surprising result. Nothing like it has been found elsewhere in the grammar; the device of node-counting somehow seems foreing to our formalisms. Furthermore, the glaring exception promise throws doubt on its correctness; it seems no accident that the meaning of promise correlates with its formal behavior under complement subject deletion». Jackendoff, Semantic Interpretation in Generative Grammar, (1972:212).

Pero con ese principio no sólo no lograron describir todos los verbos sino que la condición necesaria para la deleción de la NP con función de sujeto del infinitivo establecía que ésta debía ser idéntica a una NP de la frase principal (véase frase (2)).

Para comprobar esta condición, aportaremos sólo un ejemplo del alemán, que se presta en esta ocasión. Puesto que Rosenbaum afirmó que se trataba de un principio de la Gramática universal, y para ser correcto, debería ser también una propiedad del alemán. 
(3)

Der Hausbesitzer ordnete an, die Fahrräder in den Keller zu stellen.

El casero ordenó que se metieran las bicibletas en el sótano.

Por supuesto tampoco se cumple esta condición en todas las oraciones como demuestra el verbo anordnen. En (3) no hay ningún objeto directo que pudiera tener función de sujeto del infinitivo zu stellen. La única NP existente en esta frase, der Hausbesitzer, se excluye como posible sujeto de infinitivo, ya que debido al significado de ordenar, el sujeto, en un plano sintáctico, es aquél que ha pronunciado la orden y no a quien va dirigida, y por lo tanto no puede tener función sintáctica de sujeto del infinitivo. Este comportamiento sintáctico se debe a que anordnen, al contrario que befehlen (ordenar), no admite objeto directo alguno, dirigiéndose a quien le concierne, de modo que la condición necesaria de Rosenbaum queda anulada.

No obstante, el principio de mínima distancia se mantiene en sus rasgos esenciales, calificándolo inclūso de elemento constitutivo en la teoría del control, formulada en 1981. La posibilidad ponderada por Rosenbaum, de marcar el sujeto del infinitivo en la subcategorización léxica no se examina más.

«... if such a principle (...) is not valid, that is, if a linguistic theory does no include such a principle, the theory predicts, in efect, that the deletion of initial noun phrases in complement will be, for all languages unsystematic, dependent upon distribution at best and upon lexical subategorization at worst, and essentially random». Rosenbaum (1970:28).

Según la innovación de la Gramática Generativa en Lectures on Government and Binding, Chomsky (1981), Chomshy hace un nuevo intento de dar sistemacidad a la problemática del sujeto del infinito en la teoría del control, sustituyendo la estructura profunda por la componente formal lógica (LF) y la NP de la frase completiva, en el plano de la estructura profunda, por la categoría vacía «PRO» o «empty element».

Si el predicado lleva la señal [-Finit], se trata de un verbo de control, que por consiguiente, exige un complemento no-finito. En ese caso la NP no se expande $\left[\alpha \rightarrow \mathrm{NP}^{\mathrm{e}}\right]$.

Otra función de la teoría del control es la de establecer que una NP de la frase completiva (que sería el antecedente) controla la NP ${ }^{\text {e }}$ («e», como ya señalamos anteriormente, significa «empty») de la frase completiva (que sería la anáfora), bajo la condición de que el antecedente comande a su anáfora. En ese caso la anáfora está ligada.

En caso de que no existiera ninguna categoría que pudiera comandar la referencia, se asignaría arbitrariamente (arb). Esta limitación fue necesaria para poder incluir frases impersonales como la siguiente:

It is unclear [what [PRO to do t]]

En lo demás mantiene el principio de mínima distancia:

«I will assume that the basic principle of control is the «Minimal Distance Principle», (...) that is, a verb with a complement assigns complement control and a verb lacking a complement assigns subject control». Chomsky (1980:33) 
Ni la revisión de la «Standard Theory» ni las críticas que surgieron hacen que Chomsky repase de nuevo su enfoque incluso 10 años después de haber sido formulado por vez primera y cuyos límites fueron reconocidos desde el comienzo.

En todo caso, nos parece importante destacar, que la Grámatica Generativa demostró que todo intento de explicar fenómenos gramaticales exclusivamente con la sintaxis, es decir, limitándose a la estructura, como es el caso de la proximidad e identidad de dos NPs, sólo resultarán erróneos, si no se parte de un enfoque que explique un comportamiento sintáctico por el significado o al menos que permita el recurso a la semántica. Jackendoff fue el primero en destacar la correlación entre sintaxis y semántica, sin embargo, su planteamiento no fue tomado en serio.

Nuestro objetivo es señalar los límites de la Gramática Generativa y la necesidad de replantearse las exigencias a una gramática, que no necesariamente tiene que ser generativa. Lo cierto es que cada hablante tiene «una» representación mental de las estructuras de su lengua materna, pero no parece probado que esas representaciones tengan alguna semejanza ni con la estructura profunda ni con las transformaciones de Chomsky.

Uno de los objetivos en un replanteamiento de la teoría gramatical debe ser explicar la composición del contenido semántico a través de las palabras, ya que todo fin de una comunicación es transmitir un mensaje. Pero para ello habrá que tener especial consideración no sólo del contenido semántico de todas las palabras sino sobre todo del predicado de la frase, como veremos en el siguiente apartado.

En este sentido expondremos una aportación sumamente provechosa de John R. Searle, elaborada dentro del marco de la filosofía del lenguaje, y cuya tesis inicial es que hablar es actuar intencionadamente. Su planteamiento del acto lingüístico ha de servirnos como base para una nueva teoría gramatical..

Evidentemente no fue el objetivo de Searle superar las insuficiencias de las distintas gramáticas que se ocuparon de las construcciones de infinitivo, sino que hay que situar su ensayo Taxonomy of Illocutionary Acts (1971/76) más bien como contribución al estudio de la teoría del acto lingüístico y ésta última en la teoría de la acción, iniciada por Austin en How to do Things with Words (1962).

Lo innovador del planteamiento de Searle, probablemente por no provenir de la lingüística, consiste precisamente en partir del significado para desarrollar y explicar propiedades sintácticas de los actos ilocutivos. Por ello, la clave de su Taxonomy of Illocutionary Acts (1971/76) es, a nuestro parecer, su afirmación de que

«basic semantic differences are likely to have syntactical consecuences». $(1971 / 76: 1)$

Searle destaca que hay que recurrir a la semántica de los distintos actos ilocutivos, lo que más adelante nos servirá tambíen como base para determinar el sujeto del infinitivo.

Searle establece 5 clases de actos ilocutivos, de los que sólo los dos siguientes son relevantes: 
a) Los actos ilocutivos directivos.

b) los actos ilocutivos comisivos.

distinguiendo ambos por su fin ilocutivo, que en caso de los directivos puede reducirse al «attempt» (...) by the speaker to get the hearer to do something». (1971/76:9).

La estructura profunda para la clase de los actos ilocutivos directivos es:

I Verb you + you Fut Vol Verb (NP) (Adverb)

El hablante del enunciado corresponde pues dentro de un plano sintáctico a aquella parte de la oración que tiene la función sintáctica del sujeto.

El oyente, en una situación comunicativa, corresponde, dentro de un plano sintáctico, a aquella parte de la oración, que tiene la función sintáctica de sujeto del infinitivo.

Esa propiedad sintáctica es válida para toda la clase de verbos que son elemento de la clase de los actos ilocutivos directivos, únicamente por su significado o su fin ilocutivo, sin necesidad de argüir con proximidad o identidad de las NPs en una frase.

Searle opone a los directivos los actos ilocutivos comisivos,

«whose point is to commit the speaker to some future course of action». $(1971 / 76: 9)$

La estructura profunda de esa clase de actos ilocutivos es por consiguiente:

I Verb (you) + I Fut Vol Verb (NP) (Adverb)

La estructura profunda refleja el significado de los actos ilocutivos de la clase de los comisivos, ya que el hablante, al enunciar el acto ilocutivo p. ej. prometer, se compromete a hacer, lo que viene especificado por el infinitivo.

A pesar de que Searle asume una estructura profunda, en la que está realizado el sujeto del infinitivo, su enfoque es más adecuado que el de la Gramática Generativa, superando a ésta, al partir de la semántica para explicar un problema aparentemente sintáctico logrando de esta manera especificar sin necesidad de recurrir a una teoría complicada el posible sujeto del infinitivo y con la ventaja de no tener ninguna clase de verbos que resulte ser una excepción. El único inconveniente que sí tendría este procedimiento es, sin embargo, que hay que recurrir al significado de cada verbo para poder averiguar el sujeto del infinitivo.

El enfoque de Searle constituye una aportación teórica importante dentro de la teoría gramatical y no sólo con respecto a la discusión de las construcciones de infinitivo, que, precisamente, llevaba estancada desde 1970, es decir, desde su comienzo, debido en parte a la intransigencia de la Gramática Generativa en cuestiones semánticas, aludidas en párrafos precedentes.

De este modo, un filósofo de la lengua, sienta las bases para una nueva etapa en la teoría gramatical, que tiene como objetivo la explicación de la semántica, y que considera la sintaxis como medio para «transportar» el significado, pero nunca como objetivo de una lingüística. 


\section{BIBLIOGRAFÍA}

Chomsky, Noam, 1978, Syntactic Structures, Den Haag, Mouton.

ChOMSKy, Noam, $1977^{2}$. Logical Structure of Linguistic Theory New York: Plenum Press.

Chomsky, Noam, 1969. Aspekte der Syntax Theorie. Frankfurt am Main: Suhrkamp Verlag.

CHOMsky, Noam, 1980. On Binding. en: Linguistic Inquiry Vol. II Number I (Winter, 1980), pág. I-46, Cambridge, Massachussets: Samuel Jay Keyser (Ed.) M. I. T. Press.

Chomsky, Noam, 1981. Lectures on Government and Binding. Dordrecht: Foris Publ.

JACKENDOFF, Ray S., 1972. Semantic Interpretation in Generative Grammar. Cambridge, Massachussetts, London, England: M. I. T. Press.

Rosenbaum, Peter S., 1970. A Principle Governing Deletion in English Sentential Complementation. en: Readings in English Transformational Grammar, Jacobs, R. / Rosenbaum, P. (Ed.) Wattham, Massachussets: Ginn and Company, S. 20-29.

SEARLE, John R., 1971/76. A Taxonomy of Illocutionary Acts. Series A Papers № 40. L. A. U. T. Trier. 
